\title{
Responsiveness of articular cartilage from normal and inflamed mouse knee joints to various growth
} factors

\author{
Pernette J Verschure, Leo A B Joosten, Peter M van der Kraan, Wim B Van den Berg
}

\begin{abstract}
Objective-Disturbed anabolic signalling might contribute to the decreased chondrocyte proteoglycan (PG) synthesis during joint inflammation. Articular cartilage obtained from mouse knee joints with experimentally-induced arthritis exhibits a state of nonresponsiveness towards stimulation of chondrocyte PG synthesis by insulin-like growth factor-1 (IGF-1). Investigations were carried out on the role of other growth factors apart from IGF-1 on regulation of chondrocyte PG synthesis under pathological conditions, that is, during repair after IL-1 exposure as well as during early and later arthritis.
\end{abstract}

Methods-Mouse patellae were obtained from normal knee joints and joints injected with IL-1 or zymosan. The patellae were cultured with basic fibroblast growth factor [bFGF], plateletderived growth factor [PDGF], epidermal growth factor [EGF] or transforming growth factor $\beta$ [TGF $\beta$ ] for 24 hours in the presence or absence of IGF-1. Chondrocyte PG synthesis was measured by ${ }^{35} \mathrm{~S}$-sulphate incorporation.

Results-In normal cartilage none of the tested growth factors elicited stimulatory effects on the chondrocyte PG synthesis as caused by IGF-1. EGF and TGF $\beta$ even caused significant inhibition of chondrocyte PG synthesis. Combination of bFGF or PDGF with IGF-1 exerted significant additional stimulation of the ${ }^{35}$ S-sulphate incorporation. IL-1 exposed cartilage displayed reactivity to IGF-1 as well as to the other growth factors similar to control cartilage. Cartilage obtained from joints with experimentally-induced arthritis exhibited a state of nonresponsiveness towards all individually tested growth factors as well as growth factor combinations.

Conclusion-Arthritis causes nonresponsiveness to stimulation of chondrocyte PG synthesis by the tested growth factors, which might be caused by a general receptor function defect.

(Ann Rheum Dis 1994; 53: 455-460)

In inflammatory joint diseases, such as rheumatoid arthritis (RA), the articular cartilage is severely destroyed. ${ }^{1}{ }^{2}$ Disturbance of the equilibrium between synthesis and degradation of matrix proteoglycans (PGs) is thought to be the main cause of this deterioration of the cartilage matrix. Investigation of the phenomenon of PG synthesis inhibition has mainly been focused on the search for suppressive mediators produced by the inflamed synovium, such as interleukin-1 $(\text { IL-1 })^{3-6}$ and tumour necrosis factor $\alpha .^{78}$ These cytokines are highly potent in effecting inhibition of PG synthesis as well as causing degradation of the cartilage. Conversely, a lack of anabolic signalling ${ }^{9}$ might contribute to decreased chondrocyte PG synthesis during joint inflammation.

The precise nature by which growth factors regulate chondrocyte metabolism in normal and pathophysiological conditions is not fully understood. Insulin-like growth factor-1 [IGF1] is the most important anabolic factor for PG synthesis in normal articular cartilage. ${ }^{10-14}$ Most information on this subject is gained from studies with isolated chondrocytes or cartilage explants. Variability in responsiveness of cartilage upon culture with various growth factors, that is, basic fibroblast growth factor [bFGF], platelet-derived growth factor [PDGF], epidermal growth factor [EGF], transforming growth factor $\beta$ [TGF $\beta]$ and IGF-1 is thought to rely on differences in culture conditions or cartilage origin and species. ${ }^{15-32}$ However, little information is available about the role of these growth factors in the metabolism of chondrocytes in anatomically intact articular cartilage. ${ }^{29}$ Even less has been reported about the function of growth factors on chondrocyte PG synthesis of articular cartilage from diseased knee joints.

We previously demonstrated that IGF-1 is able to maintain PG synthesis of murine anatomically intact cartilage during culture at the in vivo level. Conversely, during experimentally-induced arthritis articular cartilage exhibited a state of non-responsiveness towards stimulation of chondrocyte PG synthesis by IGF-1. ${ }^{9}$ The aim of the present study was to investigate whether arthritic cartilage also displays nonresponsiveness to other growth factors apart from IGF-1, or whether these growth factors can compensate for IGF-1 under pathological conditions. We therefore studied the effects of bFGF, PDGF, $\mathrm{EGF}$ and TGF $\beta$, in the presence or absence of IGF-1 on the in vitro chondrocyte PG synthesis of intact normal murine patellar cartilage as well as during repair after IL-1 exposure and during early and later arthritis. 


\section{Materials and methods}

ANIMALS

Eight to 12 week old female C57 black/6 mice were used for this study. They were maintained under routine laboratory conditions. The animals were kept in boxes with sawdust bedding in an airconditioned room $\left(21-22^{\circ} \mathrm{C}\right.$, relative humidity $60 \%$ ) and a 12 hour lightdark cycle. The mice were fed a standard commercial pellet diet (RHM, Hope Farms, The Netherlands) and given acidified tap water ad libitum.

INTRA-ARTICULAR IL-1 INJECTIONS

Murine recombinant IL-1 (300 U) (kindly supplied by Dr I G Otterness, Pfizer Central Research, Groten, CT, USA) was injected in a volume of $6 \mu$ linto the joint cavity of the right knee. The left contralateral joint received an equal volume of phosphate buffered saline (PBS). The biological activity of IL-1 was determined using the lymphocyte-activation factor assay (LAF). Consistently, $10-40 \mathrm{pg} / \mathrm{ml}$ IL-1 corresponded to 1 Unit biological activity. The IL-1 batch was stored at $-20^{\circ} \mathrm{C}$, and showed constant LAF activity over the period studied.

INDUCTION OF ARTHRITIS

Arthritis was induced in the right knee joint of the animals by intra-articular injection of 180 $\mu \mathrm{g}$ sterilised zymosan [Sigma, St Louis, MO, USA] in pyrogen-free phosphate buffered saline. This model, which is characterised by a polymorphonuclear cell rich infiltrate and by cartilage damage in the acute phase (days 1-4), has been described in earlier studies. ${ }^{933}$

CARTILAGE CULTURES

Two and five days after induction of zymosaninduced arthritis as well as one, two, three and four days after injecting IL-1, mice were killed by cervical dislocation. Whole patellae were dissected from the right arthritic and left control knee joints, with a standard amount of surrounding tissue. The fully intact cartilage $^{3334}$ was subsequently used for radiosulphate incorporation studies. Radiosulphate incorporation was measured either directly at $\mathrm{t}=0$, reflecting the in vivo situation, or after culture for 24 hours. Patellae were cultured in medium, that is, RPMI 1640 (Flow Laboratories Irvine, Scotland, UK) supplemented with $2 \mathrm{mM}$ glutamine, $40 \mathrm{mg} / \mathrm{ml}$ gentamycin, $0 \cdot 1 \%$ ultrapure bovine serum albumin (Sigma). In addition, patellae were cultured in medium containing bFGF, PDGF or EGF in concentrations ranging from 0.015 $\mu \mathrm{g} / \mathrm{ml}$ to $2 \mu \mathrm{g} / \mathrm{ml}$, or TGF $\beta 2 \cdot 5-20 \mathrm{ng} / \mathrm{ml}$ (Serva, Germany), in the absence or presence of $0.25 \mu \mathrm{g} / \mathrm{ml}$ recombinant human IGF-1 (Boehringer Mannheim, Germany). The concentration range for bFGF, PDGF and EGF is based on analogy with known doseresponse effects of IGF-1 on chondrocyte PG synthesis of anatomically intact murine articular cartilage. The concentrations used for
TGF $\beta$ are analogous to those used by van der Kraan et al (1992) ${ }^{29}$ in studies with murine articular cartilage. All incubations were performed for 24 hours in 24 well cluster dishes at $37^{\circ} \mathrm{C}$ in a humidified atmosphere containing $5 \%$ carbon dioxide.

IN VITRO RADIOSULPHATE INCORPORATION Chondrocyte PG synthesis in patellar cartilage was measured by the incorporation of ${ }^{35} \mathrm{~S}$-sulphate as previously described. ${ }^{34}$ Briefly, cartilage was incubated in RPMI 1640 medium with $1.48 \quad \mathrm{MBq}{ }^{35} \mathrm{~S}$-sulphate $\left[\mathrm{Na}_{2}{ }^{35} \mathrm{SO}_{4}\right.$, Amersham, Buckinghamshire, $\mathrm{UK}$ ] for three hours at $37^{\circ} \mathrm{C}$. Thereafter, the tissue was washed three times in physiological saline, to remove non-incorporated radio label. Subsequently, patellae were fixed in $4 \%$ buffered formaldehyde and decalcified in 5\% formic acid for three hours. The patellar cartilage was then removed from the surrounding tissue and digested in lumasolve (Perstop Analytical, Oud-Beyerland, The Netherlands) at $60^{\circ} \mathrm{C}$. The quantity of incorporated radioactive sulphate was assayed by liquid scintillation counting. ${ }^{33}$

Statistical evaluation of the experiments was tested by one way analysis of variance in combination with Student's $t$ test. A p value of less than $5 \%$ was considered to be significant.

\section{Results}

EFFECT OF GROWTH FACTORS ON NORMAL CARTILAGE

PG synthesis was measured in patellar cartilage by three hours culture with ${ }^{35} \mathrm{~S}$-sulphate, either directly after isolation of the cartilage, or after a 24 hour culture period. After culture in medium without serum or growth factors, the PG synthesis rapidly declined. Recombinant human IGF-1 [0.5-2 $\mu \mathrm{g} / \mathrm{ml}]$ dose-dependent stimulated PG synthesis of normal murine patellar cartilage. ${ }^{9}$ During our study we used $0.25 \mu \mathrm{g} / \mathrm{ml} \mathrm{IGF-1} \mathrm{which} \mathrm{is} \mathrm{close} \mathrm{to} \mathrm{the} \mathrm{murine}$ plasma level. ${ }^{35}$ At this IGF-1 concentration PG synthesis could easily be maintained at the in vivo level (fig 1 ).

It was tested whether other growth factors, that is bFGF, PDGF, EGF or TGF $\beta$, could exert stimulatory effects comparable to stimulation by IGF-1. The growth factors bFGF, PDGF and EGF were tested in a concentration range of $0.015-2.0 \mu \mathrm{g} / \mathrm{ml}$ whereas TGF $\beta$ was examined in the range $0.0025-0.02 \mu \mathrm{g} / \mathrm{ml}$. None of the growth factors tested individually were able to induce stimulation of the ${ }^{35} \mathrm{~S}$-sulphate incorporation as observed for IGF-1, whereas EGF and TGF $\beta$ significantly inhibited the chondrocyte PG synthesis (fig 2). bFGF and PDGF, 0.06 and $0.125 \mu \mathrm{g} / \mathrm{ml}$, caused a slight but not significant stimulation compared with the PG synthesis after culture in medium without serum or growth factors (fig 2).

Furthermore, we studied the effects of the same set of growth factors in combination with $0.25 \mu \mathrm{g} / \mathrm{ml}$ IGF-1. As shown in fig $2, \mathrm{bFGF}$ and PDGF at $0 \cdot 125 \mu \mathrm{g} / \mathrm{ml}$ in the presence of 


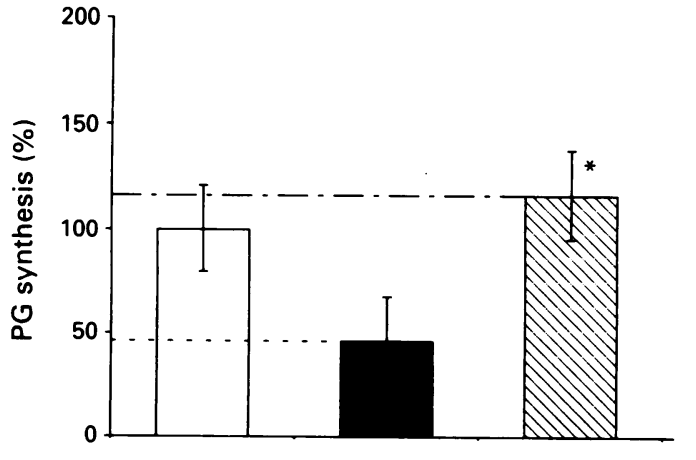

IGF-1

$t=24 h$

Figure 1 In vitro response of normal murine cartilage to $I G F-1$. Chondrocyte $P G$ synthesis of normal murine patellar cartilage was determined by ${ }^{35} \mathrm{~S}$-sulphate incorporation either immediately after isolation of the patellae $[t=0]$ or after 24 hours culture with medium alone or medium containing IGF-1 $[0.25 \mu \mathrm{g} / \mathrm{ml}]$. The experiments were performed three times, in each experiment five patellae were measured; shown are the pooled data of these three experiments, mean (SEM), expressed as percentage of the control value at $t=0 .{ }^{*} p<0.05$ compared with radiosulphate incorporation after culture with medium alone.

$0.25 \mu \mathrm{g} / \mathrm{ml} \mathrm{IGF-1} \mathrm{were} \mathrm{able} \mathrm{to} \mathrm{significantly}$ stimulate articular cartilage PG synthesis above stimulation caused by IGF-1. In contrast, the combination of EGF or TGF $\beta$ with IGF-1 did not elicit such stimulatory effects, but caused a considerable decrease in chondrocyte PG synthesis (fig 2).
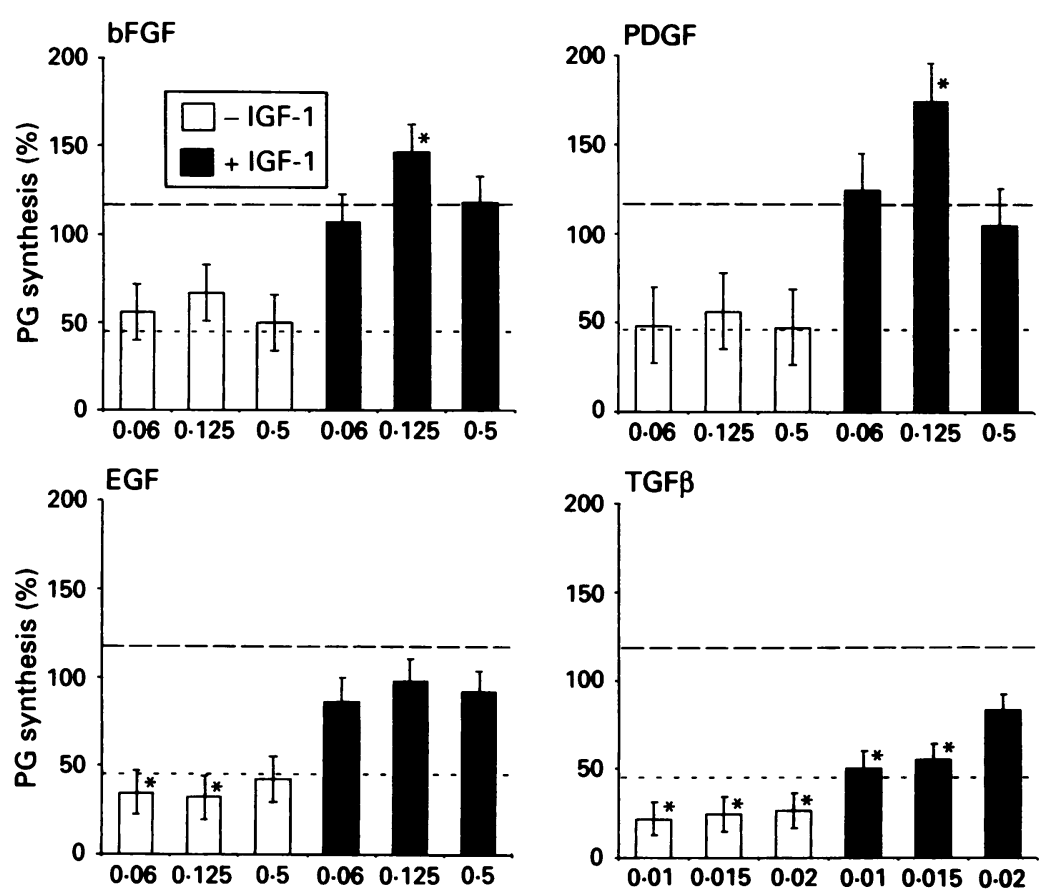

Figure 2 Effect of growth factors on $P G$ synthesis of normal murine articular cartilage. Murine normal patellae were cultured for 24 hours with $b F G F, P D G F, E G F$ in a concentration range of $0.015-2.0 \mu \mathrm{g} / \mathrm{ml}$ whereas $T G F \beta$ was examined in the range $0.0025-0.02 \mu \mathrm{g} / \mathrm{ml}$. Shown are bFGF [A], PDGF [B] or EGF [C] (concentrations $0.06-0.125-0.5 \mu \mathrm{g} / \mathrm{ml})$ or TGF $[\mathrm{D}](0.015-2.0 \mu \mathrm{g} / \mathrm{ml})$ in the absence or presence of $I G F-1[0 \cdot 25 \mu \mathrm{g} / \mathrm{ml}]$. The patella cultures were pulse-labelled with ${ }^{35} \mathrm{~S}$-sulphate for three hours to measure PG synthesis. PG synthesis is expressed as \% of ex vivo PG synthesis of the cartilage immediately after dissection. The experiments were performed three times, in each experiment five patellae were measured; shown are the pooled data of these three experiments, mean (SEM), expressed as percentage of the control value at $t=0 .{ }^{*} p<0.05$ versus radiosulphate incorporation after culture with IGF-1.

.... Chondrocyte $P G$ synthesis after culture for 24 hours with medium only $-{ }_{-}$: Chondrocyte PG synthesis after culture for 24 hours with IGF-1 [0.25 $\left.\mu \mathrm{g} / \mathrm{ml}\right]$

EFFECT OF GROWTH FACTORS ON CARTILAGE FROM IL-1 INJECTED JOINTS

Within 24 hours after intra-articular injection of IL-1 into the mouse knee joint, ex vivo chondrocyte PG synthesis was reduced to about $50 \%$ of chondrocyte PG synthesis in cartilage from the control joint (fig 3). Three days after injecting IL-1 the PG synthesis was almost restored to the level of the control joint and after four days an overshoot in PG synthesis was observed.

At different stages after IL-1 injection, culture of the cartilage with IGF-1 induced significant stimulation of chondrocyte PG synthesis (fig 3). Though at day four after IL-1 injection ex vivo PG synthesis was already at such a high level that no further stimulation by IGF-1 could be effected. Since in normal cartilage bFGF and PDGF in combination with IGF-1 were able to significantly stimulate chondrocyte PG synthesis, we studied the effects of bFGF and PDGF in the presence and absence of IGF-1 on PG synthesis of IL-1 exposed cartilage. In line with findings on normal cartilage bFGF or PDGF individually caused no stimulation of chondrocyte PG synthesis in IL-1 exposed cartilage (fig 4). Likewise, the combination of bFGF or PDGF with IGF-1 stimulated PG synthesis on top of the stimulation caused by IGF-1 alone. Moreover, combination of the three growth factors did not cause further stimulation of ${ }^{35} \mathrm{~S}$-sulphate incorporation in IL-1 exposed cartilage compared with cartilage cultured with bFGF or PDGF with IGF-1. The same observations were noted when these experiments were performed at days 2 and 4 after IL-1 injection (data not shown).

EFFECT OF GROWTH FACTORS ON ARTHRITIC CARTILAGE

At day two after zymosan-induced arthritis, ex vivo $P G$ synthesis in arthritic cartilage was considerably reduced $(50 \%)$. After culture of the arthritic cartilage in medium without serum or growth factors the chondrocyte PG

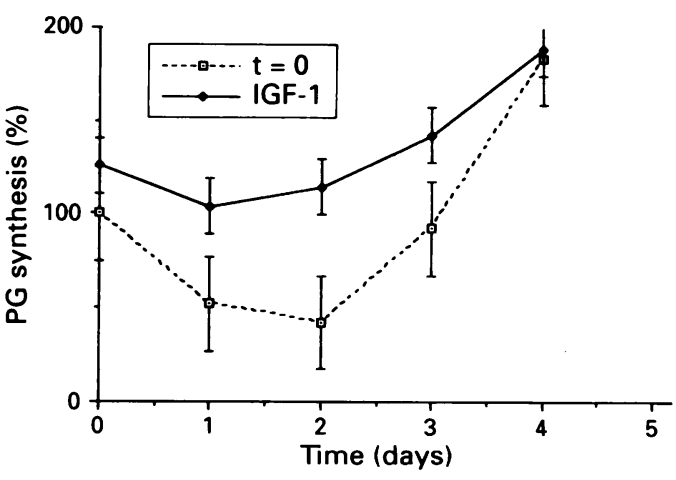

Figure 3 In vitro response of patellar cartilage from IL-1 injected knee joints. Chondrocyte PG synthesis of patellar cartilage at one, two, thee and four days after a single intraarticular IL-1 injection [300 U] was determined by ${ }^{35} S-$ sulphate incorporation either immediately after isolation of the patellae $[t=0]$ or after 24 hours culture in medium containing $0 \cdot 25 \mu \mathrm{g} / \mathrm{ml} I G F-1$. The experiments were performed three times, in each experiment five patellae were measured; shown are the pooled data of these three experiments, mean (SEM), expressed as percentage of the control value at $t=0$. 

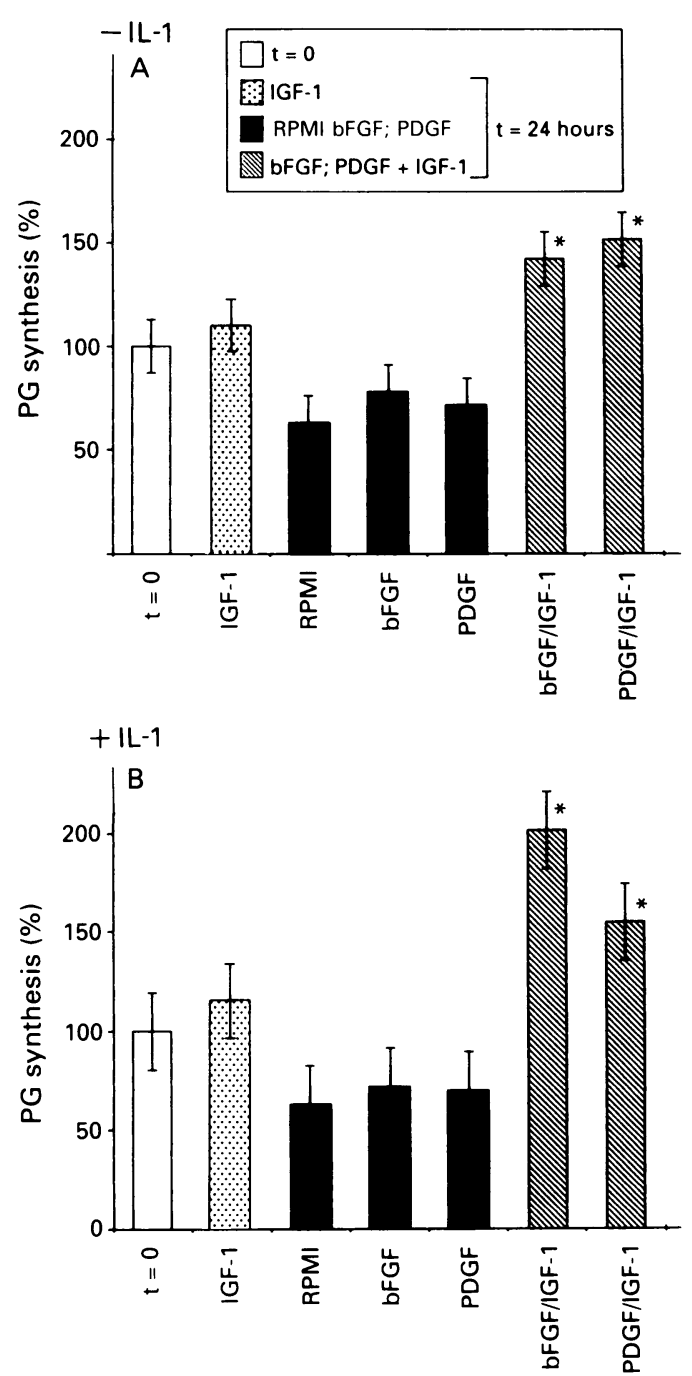

Figure 4 Effect of growth factors on ex vivo recovery of $P G$ synthesis of murine articular cartilage from $I L-1$ injected knee joints. Three days after a single intra-articular injection of IL-1 [300 U] mouse patellae were isolated from the left control joint $(A)$ and IL-1 injected inflamed joint (B). The patellae were cultured for 24 hours with medium, bFGF or PDGF $(0 \cdot 125 \mu \mathrm{g} / \mathrm{ml})$ in the absence or presence of IGF-1 [0.25 $\mu \mathrm{g} / \mathrm{ml}]$ and further treated as described in the fig 2 legend. $P G$ synthesis is expressed as \% of $P G$ synthesis of the normal cartilage immediately after dissection. At the start of the culture ${ }^{35} S$-sulphate incorporation in cartilage from the IL-1 injected joint was $497+86(\mathrm{cpm})$ and in the control cartilage $569+40$

(cpm). The experiments were performed three times, in each experiment five patellae were measured; shown are the pooled data of these three experiments, mean (SEM), expressed as percentage of the control value at $t=0$. $\star_{p}<0.05$ versus radiosulphate incorporation after culture with IGF-1.

synthesis stayed at the same low level as the ex vivo synthesis. Figure 5 shows that the addition of $0.25 \mu \mathrm{g} / \mathrm{ml} \mathrm{IGF-1} \mathrm{did} \mathrm{not} \mathrm{significantly}$ stimulate PG synthesis. We then investigated whether other growth factors apart from IGF-1 could stimulate PG synthesis of chondrocytes during the acute phase of experimentallyinduced arthritis. Figure 5A shows that after a 24 hour culture period with bFGF, PDGF, EGF $(0.125 \mu \mathrm{g} / \mathrm{ml})$ or TGF $\beta(0.015 \mu \mathrm{g} / \mathrm{ml})$ the rate of radiosulphate incorporation remains at a low level. These data indicate that during joint inflammation none of the tested growth factors could significantly stimulate PG synthesis. In addition, when the growth factors were added in combination with IGF-1 no
A Zymosan-induced arthritis (day 2)

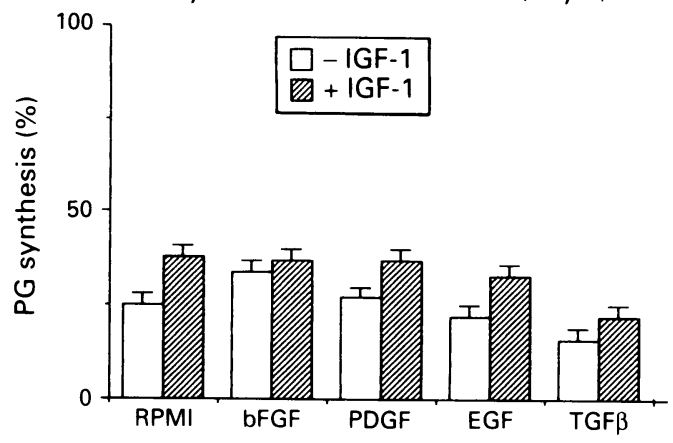

B Zymosan-induced arthritis (day 5)

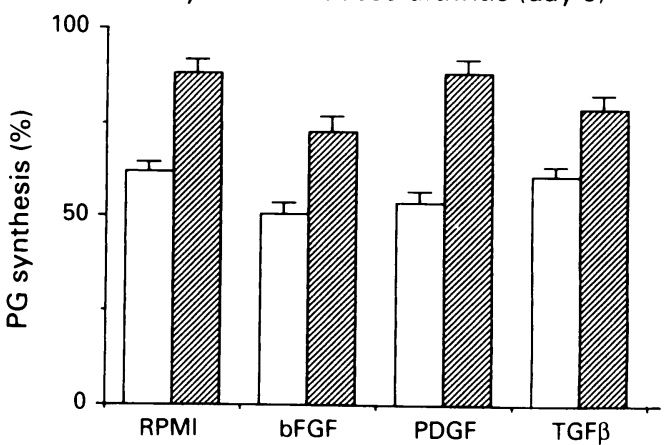

Figure 5 Effect of growth factors on $P G$ synthesis of murine arthritic articular cartilage. At day two $[A]$ and day five [B] after zymosan-induced arthritis mouse patellae were dissected from the right arthritic and left control knee joint. The patellae were cultured for 24 hours with medium, $b F G F, P D G F$, or EGF $(0 \cdot 125 \mu \mathrm{g} / \mathrm{ml})$ or TGF $\beta(0 \cdot 015$ $\mu \mathrm{g} / \mathrm{ml})$ in the absence or presence of IGF-1 $[0 \cdot 25 \mu \mathrm{g} / \mathrm{ml}]$ and further treated as described in the fig 2 legend. The ex vivo $P G$ synthesis during the actue phase of arthritis (day 2) or a later phase of arthritis (day 5) was respectively $50 \%$ and $80 \%$ compared with $P G$ synthesis of the control

cartilage. The experiments were performed three times, in each experiment five patellae were measured; shown are the pooled data of these three experiments, mean (SEM), expressed as percentage of the control value at $t=0$. ${ }_{*} p<0.05$ versus radiosulphate incorporation after culture with IGF-1.

stimulatory effect on chondrocyte PG synthesis was observed either (fig 5A).

Five days after arthritis induction, ex vivo PG synthesis was already restored to $80 \%$ of the level observed in control cartilage (data not shown). As shown in figure $5 \mathrm{~B}$, after culture in the presence of IGF-1 stimulation of PG synthesis was almost restored to the level of ex vivo control cartilage PG synthesis. Culture with bFGF, PDGF, or TGF $\beta$ did not result in enhanced ${ }^{35} \mathrm{~S}$-sulphate incorporation. Furthermore, combination of bFGF, PDGF or TGF $\beta$ with IGF-1 did not induce stimulation above effects found after culture with IGF-1 alone.

\section{Discussion}

At present there is substantial evidence indicating that one of the mechanisms leading to cartilage destruction in inflammatory joint diseases is inhibition of chondrocyte PG synthesis. ${ }^{33}{ }^{36} \mathrm{It}$ is still not precisely known how this inhibition is effected, although possible evidence is accumulating that IL-1 plays a crucial role in this process. ${ }^{3-6}{ }^{37}$ We previously suggested that inhibition of chondrocyte metabolism might also be caused by inadequate anabolic regulation of PG 
synthesis. ${ }^{9}$ In the present study we investigated the role of other growth factors apart from IGF-1 in the regulation of chondrocyte PG synthesis in cartilage from normal and arthritic mouse knee joints.

It is generally accepted that IGF-1 is an important growth factor in the regulation of normal cartilage PG synthesis. ${ }^{10-14}$ Our data indicate that in normal cartilage none of the individually tested growth factors possessed the capacity to induce stimulatory effects on chondrocyte PG synthesis similar to stimulation by IGF-1. However, in combination with IGF-1, bFGF and PDGF induced pronounced enhancement of PG synthesis. In contrast, EGF and TGF $\beta$ even significantly decreased chondrocyte PG synthesis. Controversial results have been described concerning the ability of various growth factors or combinations of growth factors to stimulate articular cartilage matrix synthesis, depending either upon difference in culture conditions or in cartilage origin and species. ${ }^{21}{ }^{30-32}$ bFGF has been shown to considerably stimulate PG synthesis of actively growing chondrocytes ${ }^{20} 313238$ whereas other studies showed that bFGF as well as EGF alone did not, or only moderately, effect PG synthesis of differentiated chondrocytes in culture. Likewise, Wroblewski and Edwall showed that PDGF had no appreciable effect on PG synthesis of rat differentiated chondrocytes, whereas for confluent chondrocyte cultures it has been reported that PDGF stimulates PG synthesis to the same extent as IGF-1. ${ }^{17} 2139$ We show, however, that both under normal and pathological physiological conditions exogenous exposure of intact cartilage to bFGF, PDGF, EGF or TGF $\beta$ did not elicit stimulation of the chondrocyte PG synthesis within 24 hours.

Our findings that TGF $\beta$ significantly inhibited the chondrocyte PG synthesis of intact patellar cartilage agree with previous studies. ${ }^{29}$ It was shown that for intact mouse patellar cartilage neutralisation of endogenous produced TGF $\beta$ with a specific anti-TGF $\beta$ antibody caused stimulation of PG synthesis, indicating that TGF $\beta$ itself inhibits $P G$ synthesis. However, other studies described either minimal effects of TGF $\beta$ on PG synthesis of intact articular cartilage during short term culture, ${ }^{4041}$ or stimulation of PG synthesis after prolonged incubation in basal medium supplemented with TGF $\beta$, but not in medium containing insulin or IGF-1.41 42 Moreover, the effects of TGF $\beta$ on isolated chondrocytes are contradictory. ${ }^{17} 22-25272830$ Recently we have shown that the response of isolated chondrocytes to TGF $\beta$ correlates with the size of the TGF $\beta$ type II receptor. ${ }^{43}$ It is tempting to speculate that changes in chondrocyte phenotype cause differences in growth factor receptor expression and chondrocyte responsiveness.

During the acute phase of arthritis, chondrocytes from the arthritic joint did not respond to IGF-1 nor to one of the other growth factors, either individually tested or in combination with IGF-1. Schalkwijk et $a l^{9}$ suggested that the inflammatory response in the arthritic joint might have caused IGF-1 non-responsiveness by inactivation or downregulation of the IGF-1 receptor. Our results do not indicate that the inflammatory process in the joint might simultaneously induce an upregulation for other growth factors. It may be suggested that chondrocytes from the diseased joint display a general receptor function defect.

Moreover, we wondered if and which growth factors are concerned with repair processes after IL-1 injection in the mouse knee joint. Our data show that in IL-1 exposed cartilage IGF-1 stimulation of PG synthesis equalled synthesis of the control cartilage, cultured in the same way. This may imply that the phenomenon of IGF-1 non-responsiveness as observed during experimentally-induced arthritis, is not only dependent upon IL1-induced PG synthesis inhibition. Inflammatory processes in the joint during arthritis, leading to PG synthesis inhibition, seem to be more complex than processes induced by IL-1 injection. However, anti-IL-1 treatment of arthritic mice leads to restoration of PG synthesis and almost normal IGF responsiveness, indicating that IL-1 is indirectly involved in the process of IGF-1 nonresponsiveness. ${ }^{37}$ IL-1 exposed cartilage displayed similar reactivity to stimulation by growth factors apart from IGF-1 as control cartilage. In contrast, Harvey et $a l^{44}$ reported that during the repair phase after IL-1 induced PG depletion, culture with PDGF induced an overshoot in PG synthesis.

It is widely speculated that growth factors might play a role in repair processes during degenerative joint diseases. ${ }^{18} 22444647$ We have recently described that multiple intra-articular TGF $\beta$ injections into the mouse knee joint induced strong, longlasting stimulation of chondrocyte PG synthesis. ${ }^{48}$ Moreover, TGF $\beta$ injection could counteract IL-1 induced effects. ${ }^{41}$ However, the results obtained in the present study indicate that in contrast to normal and IL-1 exposed cartilage, cartilage derived from joints with experimentally-induced arthritis shows a general state of nonresponsiveness towards growth factor stimulation. These findings indicate that before anabolic growth factors are considered to be used as therapeutic agents to stimulate repair, chondrocyte responsiveness during various pathological stages need to be further investigated.

In conclusion, exogeneous exposure of normal intact mouse articular cartilage to bFGF, PDGF, EGF or TGF $\beta$ did: 1) not elicit stimulatory effects on chondrocyte PG synthesis, as caused by IGF-1;2) EGF and TGF $\beta$ even caused a significant inhibition of chondrocyte PG synthesis; 3) In combination with IGF$1, \mathrm{bFGF}$ and PDGF exerted significant additional stimulation of chondrocyte PG synthesis; 4) IL-1-induced inflammation did not cause IGF-1 non-responsiveness; 5) IL-1 exposed cartilage showed similar reactivity to growth factor stimulation as found in control cartilage; 6) During the acute or later phases of arthritis, cartilage showed a state of nonresponsiveness towards all individually tested 
growth factors as well as 7) to combination of growth factors with IGF-1. Inhibition of PG synthesis during experimentally-induced arthritis, seems to be more complex than the processes caused by injecting IL-1 into the knee joint. It is tempting to speculate that arthritis causes a non-responsive state to chondrocyte growth factor stimulation, which may be affected by a general receptor function defect.

1 Zvaifler N J. Pathogenesis of the joint disease of rheumatoid arthritis. Am f Med 1983; 75: suppl 3 .

2 Krane S M, Amento E P, Goldring S R. Cellular interactions in tissue breakdown in rheumatoid arthritis. Advances in Inflammation Research 1986; 51: 1-12.

3 Van de Loo A A J, Van den Berg W B. Effects of murine recombinant IL-1 on synovial joints in mice: quantification of patellar cartilage metabolism and joint inflammation. Ann Rheum Dis 1990; 49: 238-45.

4 Verschure P J, Van Noorden C J F. The effects of interleukin-1 on articular cartilage destruction as observed in arthritic diseases, and its therapeutic control. Clin Exp Rheum 1990; 8: 303-13.

5 Bentzen K, Petersen J, Halkjaer-Kristensen J, IngemannHansen T. Interleukin-1-like activities in synovial fluids of patients with rheumatoid arthritis and traumatic synovitis. Rheum Int 1985; 5: 79-82.

6 Benton H P, Tyler J A. Inhibition of cartilage proteoglycan synthesis by interleukin-1. Biochem Biophys Res Commun 1988; 145: 421-8.

7 Saxne T, Palladino M A, Heunegard Jr D, Talal N, Wollheim F A. Detection of tumor necrosis factor $\beta$ in rheumatoid arthritis synovial fluid and serum. Arthr Rheum 1988; 31: 1041-5.

8 Saklatvala J. Tumor necrosis factor- $\alpha$ stimulates resorption and inhibits synthesis of PG in cartilage. Nature 1987; 332: 547.

9 Schalkwijk J, Joosten L A B, Van den Berg W B, Van de Putte L B A. Chondrocyte nonresponsiveness to insulinlike growth factor 1 in experimental arthritis. Arthr Rheum 1989; 32: 894-900.

10 McQuillan D J, Handley C J, Campbell M A, Bolis S, Milway V E, Herington A C. Stimulation of proteoglycan Mynthesis by serum and insulin-like growth factor-1 in synthesis by serum and insulin-like growth factor-1 in
cultured bovine articular cartilage. Biochem $\mathcal{f} 1986 ; 240$ : cultured

11 Schalkwijk J, Joosten L A B, Van den Berg W B, Wijk J J, Van de Putte L B A. Insulin-like growth factor stimulation of chondrocyte proteoglycan synthesis by human synovial fluid. Arthr Rheum 1989; 32: 66-71.

12 Luyten F P, Hacall V C, Nissley S P, Morales T I, Reddi A H. Insulin-like growth factors maintain steady-state metabolism of proteoglycans in bovine articular cartilage explants. Arch Biochem Biophys 1988; 267: 416-25.

13 Hascall V C, Handley C J, McQuillan D J, Hascall G K, Robinson H C, Lowther D A. The effect of serum on biosynthesis of proteoglycans by bovine articular cartilage in culture. Arch Biochem Biophys 1983; 224: 206-23.

14 Tyler J A. Insulin-like growth factor 1 can decrease degradation and promote synthesis of proteoglycan in cartilage exposed to cytokines. Biochem f 1989; 260: 543-48.

15 Kato Y, Hiraki Y, Inoue H, Kinoshita M, Yutani Y, Suzuki F. Differential and synergistic actions of somatomedinlike growth factors, fibroblast growth factor and epidermal growth factor in rabbit costal chondrocytes. Eur $\mathcal{f}$ Biochem 1983; 129: 685-90

16 Hiraki Y, Yutani Y, Takigawa M, Kato Y, Suzuki F. Differential effects of parathyroid hormone and somatomedin-like growth factors on the sizes of
proteoglycan monomers and their synthesis in rabbit costal chondrocytes in culture. Biochim Biophys Acta 1985; 845: 445-53.

17 Hiraki Y, Inoue H, Hirai R, Kato Y, Suzuki F. Effect of transforming growth factor $\beta$ on cell proliferation and
glycosaminoglycan synthesis by rabbit growth-plate glycosaminoglycan synthesis by rabbit growth-plate
chondrocytes in culture. Biochim Biophys Acta 1988; 969: 91-9.

18 Böhme K, Conscience-Egli M, Tschan T, Winterhalter K $\mathrm{H}$. Induction of proliferation or hypertrophy of chondrocytes in serum-free culture: The role of insulinlike growth factor-1, insulin, or thyroxine. $\mathcal{f}$ Cell Biol 1992; 116: 1035-42.

19 Wroblewski J, Edwall C. PDGF BB stimulates proliferation and differentiation in cultured chondrocytes from rat rib growth plate. Cell Biol Int Reports 1992; 16: 133-44.

20 Kato Y, Gospodarowicz D. Sulfated proteoglycan synthesis by confluent cultures of rabbit costal chondrocytes. $\mathcal{F} \mathrm{Cell}$ Biol 1985; 100: 477-85.

21 Inoue H, Kato Y, Iwamoto M, Hiraki Y, Sakuda M, Suzuki F. Stimulation of cartilage-matrix proteoglycan synthesis by morphologically transformed chondrocytes grown in the presence of fibroblast growth factor and transforming the presence of fibroblast growth factor and transform
growth factor-beta. $\mathcal{F}$ Cell Physiol 1989; 138: 329-37.

22 Redini F, Daireaux M, Mauviel A, Galera P, Loyau G, Pujo J-P. Characterization of proteoglycans synthesized by rabbit articular chondrocytes in response to transformin growth factor- $\beta$ (TGF- $\beta$ ). Biochim Biophys Acta 1991; 1093: 196-206.

23 O'Keefe R J, Puzas J E, Brand J S, Rosier R N. Effects of transforming growth factor- $\beta$ on matrix synthesis by chick growth plate chondrocytes. Endocrinology 1988; 122. 2953-61.

24 Redini F, Galera P, Mauviel A, Loyau G, Pujol J-P. Transforming growth factor $\beta$ stimulates collagen and glycosaminoglycan biosynthesis in cultured rabbit articular chondrocytes. FEBS Letters 1988; 234: 172-6.

25 Morales T I, Roberts A B. Transforming growth factor $\beta$ regulates the metabolism of proteoglycans in bovin cartilage organ cultures. F Biol Chem 1988; 263 12828-831.

26 Morales T I, Joyce M E, Sobel M E, Danielpour D, Roberts A B. Transforming growth factor- $\beta$ in calf articula cartilage organ cultures: Synthesis and distribution. $\mathrm{ArCh}$ Biochem Biophys 1991; 288: 397-405.

27 Skantze K A, Brinckerhof C E, Collier J P. Use of agarose culture to measure the effect of transforming growth factor $\beta$ and epidermal growth factor on rabbit articular chondrocytes. Cancer Res 1984; 45: 4416-21.

28 Rosier R N, O'Keefe R J, Crabb I D, Puzas J E, Brand J $\mathrm{S}$. Articular and growth plate chondrocytes exhibit markedly different metabolic responses to growth plate factors (abstract). I Bone Miner Res 1988; S185: 468

29 Van der Kraan P M, Vitters E L, Van den Berg W B. Inhibition of proteoglycan synthesis by transforming growth factor $\beta$ in anatomically intact articular cartilage of murine patellae. Ann Rheum Diseases 1992; 51: 643-47.

30 Van der Kraan P, Vitters E, Van den Berg W B. Differential effect of transforming growth factor $\beta$ on freshly isolated and cultured articular chondrocytes. I Rheumatol 1992; 19: $140-5$.

31 Hiraki Y, Inoue $\mathrm{H}$, Asada A, Suzuki F. Differential modulation of growth and phenotypic expression of chondrocytes in sparse and confluent cultures by growth factors in cartilage. F Bone Mineral Res 1990; 10: 1077-85.

32 Iwamoto M, Shimazu A, Nakashima K, Suzuki F, Kato Y. Reduction in basic fibroblast growth factor receptor is coupled with terminal differentiation of chondrocytes. $f$ Biol Chem 1991; 266: 461-7.

33 Van den Berg W B, Kruijsen M W M, Van de Putte L B A, Zwarts W A. Antigen-induced arthritis and zymosaninduced arthritis in mice: Studies in in vivo cartilage proteoglycan synthesis and chondrocyte death. $\operatorname{Br} \mathcal{F} \operatorname{Exp}$ Pathol 1981; 62: 308-16.

34 Van den Berg W B, Kruijsen $M$ W M, Van de Putte L B A. The mouse patella assay: an easy method of quantitating articular cartilage chondrocyte function in vivo and in vitro. Rheum Int 1982; 1: 165-9.

35 Van Beuningen H M, Arntz O J, Van den Berg W B. In vivo effects of interleukin-1 on articular cartilage: Prolongation of proteoglycan metabolic disturbance in old mice. Arth Rheum 1991; 34: 606-15.

36 Sandy J D, Lowther D A, Brown H L G. Antigen-induced arthritis: studies on the inhibition of proteoglyca synthesis observed in articular cartilage during short-term joint inflammation. Arthr Rheum 1980; 23: 433-47.

37 Van de Loo A A J, Arntz O J, Otterness I G, Van den Berg W B. Protection against cartilage proteoglycan synthesis inhibition by anti-interleukin 1 antibodies in experimental arthritis. F Rheum 1992; 19: 348-56.

38 Kato Y, Iwamoto $M$. Fibroblast growth factor is an inhibitor of chondrocyte terminal differentiation. $f$ Biol Chem 1990 265: 5903-909.

39 Osborn K D, Trippel S B, Mankin H J. Growth factor stimulation of adult articular cartilage. f Orthop Res 1989 7: $35-42$

40 Hardingham T E, Bayliss M T, Rayan V, Noble D P. Effect of growth factors and cytokines on proteoglycan turnove in articular cartilage. Brf Rheumatol 1992; 31 (suppl 1): $1-6$

41 Van Beuningen H M, Van der Kraan P M, Arntz O J, Van den Berg W B. Protection from interleukin-1-induced destruction of articular cartilage by transforming growth factor- $\beta_{1}$ : studies in anatomically intact cartilage in vitro and in vivo. Ann Rheum Dis 1993; 52: 185-91.

42 Burton-Wurster N, Lust G. Fibronectin and proteoglycan synthesis in long term cultures of cartilage explants in Ham's F12 supplemented with insulin and calcium: effects of the addition of TGF 3 . Arch Biochem Biophys 1990; 283: 27-33.

43 Glansbeek H L, Van der Kraan P M, Vitters E L, Van den Berg W B. Correlation of the size of type II transforming growth factor $\beta$ (TGF- $\beta$ ) receptor with TGF-b response of isolated bovine articular chondrocytes. Ann Rheum Dis 1993; 52: 812-6.

44 Harvey A K, Stack S T, Chandrasekhar S. Differential modulation of degradative and repair responses of interleukin-1 treated chondrocytes by platelet-derived growth factor. Biochem ₹ 1993; 292: 129-36.

45 Copray J C, Brower N, Prins A P, Jansen H W. Effects of polypeptide growth factors on mandibular condylar cartilage of the rat in vitro. I Biol Buccale 1988; 16: 109-17.

46 Cuevas P, Burgos J, Baird A. Basic fibroblast growth factor (FGF) promotes cartilage repair in vivo. Biochem Biophys Res Comm 1988; 156: 611-8.

47 Ogawa Y, Ksander G A Pratt B M, et al. Differences in the biological properties of transforming growth factor $-\beta$ and platelet-derived growth factor in vivo. Growth Factors 1991; 5: 57-68

48 Van Beuningen H M, Van der Kraan P M, Arntz O J, Van den Berg W B. Transforming growth factor- $\beta 1$ stimulates articular chondrocyte proteoglycan synthesis and induces osteophyte formation in the murine knee joint. Lab Invest 1994 (in press) 